\title{
Diagonal conditions in ordered spaces
}

\author{
by \\ Harold R. B e n nett (Lubbock, Tex.) and \\ David J. L u t z er (Williamsburg, Va.) \\ Dedicated to the memory of Maarten Maurice
}

\begin{abstract}
For a space $X$ and a regular uncountable cardinal $\kappa \leq|X|$ we say that $\kappa \in D(X)$ if for each $T \subset X^{2}-\Delta(X)$ with $|T|=\kappa$, there is an open neighborhood $W$ of $\Delta(X)$ such that $|T-W|=\kappa$. If $\omega_{1} \in D(X)$ then we say that $X$ has a small diagonal, and if every regular uncountable $\kappa \leq|X|$ belongs to $D(X)$ then we say that $X$ has an H-diagonal. In this paper we investigate the interplay between $D(X)$ and topological properties of $X$ in the category of generalized ordered spaces. We obtain cardinal invariant theorems and metrization theorems for such spaces, proving, for example, that a Lindelöf linearly ordered space with a small diagonal is metrizable. We give examples showing that our results are the sharpest possible, e.g., that there is a first countable, perfect, paracompact Cech-complete linearly ordered space with an H-diagonal that is not metrizable. Our example shows that a recent CH-result of Juhász and Szentmiklóssy on metrizability of compact Hausdorff spaces with small diagonals cannot be generalized beyond the class of locally compact spaces. We present examples showing the interplay of the above diagonal conditions with set theory in a natural extension of the Michael line construction.
\end{abstract}

1. Introduction. As part of his study of functions defined on product spaces, M. Hušek introduced a family of diagonal conditions in a topological space $X([\mathrm{H} 1, \mathrm{H} 2])$. For a cardinal number $\kappa$, he defined that the diagonal $\Delta(X)$ of a space $X$ is $\kappa$-inaccessible if for any $T \subset X^{2}-\Delta(X),|T|=\kappa$ implies that $|T-W|=\kappa$ for some open neighborhood $W$ of $\Delta(X)$. If the diagonal of $X$ is $\omega_{1}$-inaccessible, he said that $X$ has a small diagonal. Subsequently, these notions have played a role in metrization theory ([Zh, $\mathrm{JS}])$ and in the study of $C_{\mathrm{p}}(X)([\mathrm{A}, \mathrm{AT}])$, often for compact spaces, and

1991 Mathematics Subject Classification: Primary 54F05; 54D15; 54D30; Secondary 54E18; 54E35.

Key words and phrases: H-diagonal, small diagonal, linearly ordered topological space, generalized ordered space, cardinal invariant, metrizability, paracompact space, Cechcomplete space, p-space, Michael line, Sorgenfrey line, $\sigma$-disjoint base. 
sometimes using additional set-theoretic assumptions such as the Continuum Hypothesis $(\mathrm{CH})$.

For regular uncountable $\kappa$, Hušek's diagonal properties generalize the notion of a $G_{\delta^{-}}$-diagonal. Given the central role played by $G_{\delta^{-}}$diagonals in metrization theory for compact Hausdorff spaces and for linearly ordered spaces (see [S] and [L1], for example), it is natural to ask about the role of Hušek's diagonal conditions in metrization of such spaces, and several positive results have been obtained. The first is due to Juhász and Szentmiklóssy [JS] and the second, due to van Douwen and Lutzer, was announced in [H2] but no proof has been published until now.

(1.1) TheOrem [JS]. The Continuum Hypothesis implies that any compact Hausdorff space with a small diagonal is metrizable.

(1.2) TheOREm [H2]. Any compact linearly ordered topological space with a small diagonal is metrizable, and the same is true for any Lindelöf linearly ordered topological space with a small diagonal.

Historically, metrization theorems for compact Hausdorff spaces have often been generalized to metrization theorems for the progressively larger classes of locally compact spaces, then Cech-complete spaces, and finally the p-spaces of Arkhangel'skiı̌, often with the additional hypothesis of paracompactness $([\mathrm{Bo}, \mathrm{Ok}])$. Parallel generalizations have been found for $G_{\delta^{-}}$ and p-embedded subspaces of linearly ordered spaces ([L2]). It is, therefore, reasonable to ask whether the results in (1.1) and (1.2) can be generalized to those larger classes. The first step is very easy:

(1.3) Proposition. Suppose Y is paracompact, locally compact, and has a small diagonal.

(a) Under $C H, Y$ is metrizable.

(b) In any model of set theory, if $Y$ is a subspace of a linearly ordered space, then $Y$ is metrizable.

Proof. Such a space is locally metrizable and paracompact.

One of the goals of this paper is to show that further generalization of (1.3) to Cech-complete spaces, or to p-spaces, is not possible.

A second goal of the paper is to introduce and study a relative of Hušek's small diagonal condition.

(1.4) Definition. For a space $X$, let $D(X)$ be the set of regular uncountable cardinals $\kappa$ such that:

(a) $\kappa \leq|X|$; and

(b) the diagonal of $X$ is $\kappa$-inaccessible, i.e., if $T \subset X^{2}-\Delta(X)$ and $|T|=\kappa$, then $|T-W|=\kappa$ for some open neighborhood $W$ of $\Delta(X)$. 
(1.5) Definition. The space $X$ has an $H$-diagonal provided $D(X)=$ $\{\kappa \leq|X|: \kappa$ is a regular uncountable cardinal $\}$.

It is easy to see that if $X$ is an uncountable space with a $G_{\delta}$-diagonal then $X$ has an H-diagonal, and that if $X$ has an H-diagonal, then $X$ has a small diagonal. Examples (6.2) and (7.7) show that neither implication can be reversed. Using the notion of an $\mathrm{H}$-diagonal, we prove the following generalization of (1.2):

(1.6) Proposition. Any locally compact generalized ordered space with an H-diagonal is metrizable.

Pr o of. The key step is to prove that a generalized ordered space with an H-diagonal must be paracompact. That result appears in Section 5, below.

The possibilities for further generalization of (1.1) and (1.2) are extremely limited, as can be seen from:

(1.7) ExAmple. There is a non-metrizable, Čech-complete, linearly ordered topological space that has an H-diagonal (and hence a small diagonal).

Even though Čech-complete linearly ordered spaces with H-diagonals may fail to be metrizable, they are not without special structure, as can be seen from:

(1.8) Proposition. Let $X$ be a linearly ordered topological space with an $H$-diagonal. If $X$ is either Cech-complete or a p-space, then $X$ has a dense metrizable subspace.

Other examples in our paper illustrate the interactions of H-diagonals with various base conditions in ordered spaces, e.g.:

(1.9) EXAMPLE. If $M^{*}$ denotes the smallest linearly ordered topological space that contains the Michael line $M$ as a closed subspace, then $M^{*}$ is not metrizable, has a $\sigma$-disjoint base, and does not have an H-diagonal. However, it is undecidable in $Z F C$ whether or not $M^{*}$ has a small diagonal, and it is undecidable in $Z F C$ whether or not $c \in D\left(M^{*}\right)$.

Our paper is organized as follows. In Section 2 we present definitions from ordered space theory and remind the reader of a standard tree construction. In Section 3 we use the tree construction to establish certain cardinal function inequalities for ordered spaces with various diagonal conditions. In Section 4 we apply those results to prove Theorem (1.2) above. In Section 5 we investigate properties of generalized ordered spaces with $\mathrm{H}$ diagonals, and prove (1.6). Examples in Section 6 show the role of Hušek's diagonal conditions in familiar ordered spaces. Section 7 uses the "splitting points" technique to produce the space of Example (1.7). Section 8 uses 
another ordered space construction to investigate the role of $\mathrm{H}$-diagonals in the presence of certain base conditions, partially proving (1.9) among other results. Section 9 translates and extends certain results from Section 8 using the language of $\omega^{\omega}$ and its ordering $\leq^{*}$, and completes the proof of (1.9).

The authors would like to thank K. P. Hart for showing how to substantially shorten proofs of several results in this paper, and for pointing out results that appear in Section 9.

2. Definitions and the tree construction. Recall that a linearly ordered topological space $(L O T S)$ is a triple $(X, \mathcal{T},<)$ such that $<$ is a linear ordering of $X$ and $\mathcal{T}$ is the usual open interval topology of $<$. A subspace of a LOTS may fail to be a LOTS when equipped with the subspace topology and the restricted ordering. Subspaces of LOTS are called generalized ordered spaces (GO-spaces) and may be characterized another way: GO-spaces are triples $(X, \mathcal{T},<)$ where $<$ is a linear ordering of $X$ and $\mathcal{T}$ is a Hausdorff topology on $X$ that has a base consisting of order-convex sets. The best known GO-spaces are the Sorgenfrey line ([So]) and the Michael line ([M1]). Neither is a LOTS under any linear ordering.

Intervals in a linearly ordered set will be denoted by $] a, b[,[a, b[,[a, b]$ and ]$\leftarrow, b]$, etc. In many cases, members of the underlying linearly ordered set will be ordered pairs, and intervals will have forms such as $](a, 0),(b, 1)[$. Cardinal functions $c(X), d(X), w(X), l(X), h l(X)$ and $h d(X)$ are defined as in [E]. For any GO-space $X$, we know that $l(X) \leq h l(X)=c(X) \leq d(X)=h d(X)$ (see [L2]).

The following tree construction is standard in GO-space theory, but notation varies from one author to another. We will need the details of the construction in Section 3, so we outline it here. Our construction can be carried out in any GO-space, but it is most easily described in its historical context, namely in situations where $X$ is a GO-space having $c(X)=\kappa<d(X)$. We know that $h l(X)=c(X)=\kappa$ (but we do not know that $\kappa$ is regular or uncountable).

Let $D(0)=\bigcup\{G: G$ is open in $X$ and $|G| \leq \kappa\}$. Because $h l(X)=\kappa$, the subspace $D(0)$ can be covered by $\kappa$ open sets, each with cardinality at most $\kappa$, so that $|D(0)| \leq \kappa$. Hence $D(0)$ cannot be dense in $X$. Let $\mathcal{U}(0)$ be the family of all convex components of $X-\operatorname{cl}(D(0))$.

For each open convex $C \subset X-\operatorname{cl}(D(0))$, let $f(C) \in C$ be chosen in such a way that $C-\{f(C)\}$ has two convex components $C^{\prime}$ and $C^{\prime \prime}$, each with cardinality greater than $\kappa$, named in such a way that each point of $C^{\prime}$ is less than each point of $C^{\prime \prime}$. Such a choice is always possible, because each non-empty open set disjoint from $D(0)$ must have cardinality greater than $\kappa$. 
Suppose $\alpha<\kappa^{+}$and that for each $\beta<\alpha$ we have collections $\mathcal{U}(\beta)$ and sets $D(\beta)$ satisfying:

(a) each $\mathcal{U}(\beta)$ is a nonvoid, pairwise disjoint collection of nonvoid, convex, open subsets of $X$;

(b) if $\beta$ is a limit ordinal then $\mathcal{U}(\beta)$ is the family of convex components of $X-\operatorname{cl}(\bigcup\{D(\gamma): \gamma<\beta\})$ and $D(\beta)=\bigcup\{D(\gamma): \gamma<\beta\}$.

(c) if $\beta=\gamma+1$ is not a limit ordinal, then $D(\beta)=\{f(C): C \in \mathcal{U}(\gamma)\}$ and $\mathcal{U}(\beta)=\left\{C^{\prime}, C^{\prime \prime}: C \in \mathcal{U}(\gamma)\right\}$.

(d) for each $\beta<\alpha$, we have $|\mathcal{U}(\beta)| \leq \kappa$ and $|D(\beta)| \leq \kappa$.

(e) if $\gamma<\beta<\alpha$, then each member of $\mathcal{U}(\beta)$ is contained in a unique member of $\mathcal{U}(\gamma)$.

We now define $D(\alpha)$ and $\mathcal{U}(\alpha)$. If $\alpha$ is a limit ordinal, let $D(\alpha)=$ $\bigcup\{D(\beta): \beta<\alpha\}$. Then $|D(\alpha)| \leq \kappa$, so that $D(\alpha)$ cannot be dense in $X$. Let $\mathcal{U}(\alpha)$ be the family of all convex components of $X-\operatorname{cl}(D(\alpha))$. If $\alpha=\beta+1$ is not a limit ordinal, let $D(\alpha)=\{f(C): C \in \mathcal{U}(\beta)\}$ and let $\mathcal{U}(\alpha)=\left\{C^{\prime}, C^{\prime \prime}: C \in \mathcal{U}(\beta)\right\}$. Observe that the function $f: \mathcal{U} \rightarrow X$ is injective.

This recursive construction, carried out under the assumption that $c(X)$ $=\kappa<d(X)$, gives non-empty collections $\mathcal{U}(\alpha)$ and non-empty sets $D(\alpha)$ for each $\alpha<\kappa^{+}$. Let $\mathcal{U}=\bigcup\left\{\mathcal{U}(\alpha): \alpha<\kappa^{+}\right\}$.

(2.1) Proposition. The collection $\mathcal{U}$ is a tree and if $c(X)=\kappa<d(X)$, then $\mathcal{U}$ has no subcollection $\mathcal{V}$ with $|\mathcal{V}|=\kappa^{+}$that is linearly ordered by inclusion. In particular, no $\mathcal{V} \subset \mathcal{U}$ with $|\mathcal{V}|=\kappa^{+}$has $\bigcap \mathcal{V} \neq \emptyset$.

(2.2) Corollary. If $c(X)=\kappa<d(X)$, then the set $D=\bigcup\{D(\alpha): \alpha<$ $\left.\kappa^{+}\right\}$is dense in $X$ and has $|D|=\kappa^{+}$.

(2.3) Corollary. For any GO-space, either $d(X)=c(X)$ or else $d(X)$ $=c(X)^{+}$.

It is consistent with ZFC that a LOTS $X$ can have $c(X)=\omega$ and yet $d(X)=\omega_{1}$. Such an $X$ is a Suslin space.

3. Cardinal invariants and $D(X)$ for GO-spaces. As noted in Section 2, for any GO-space $X$, we have $l(X) \leq h l(X)=c(X) \leq d(X)=$ $h d(X)$. In this section we will investigate the interplay between those cardinal functions and the cardinals in $D(X)$.

It will sometimes be useful to have a translation of the statement " $\kappa \in$ $D(X)$ " into the language of open coverings.

(3.1) Proposition. Suppose that $X$ is any topological space and that $\kappa$ is a regular uncountable cardinal. Then $\kappa \in D(X)$ if and only if for each set $T \subset X^{2}-\Delta(X)$ having $|T|=\kappa$, there is an open cover $\mathcal{G}$ of $X$ and a subset 
$T_{0} \subset T$ such that $\left|T_{0}\right|=\kappa$ and such that if $(x, y) \in T_{0}$, then no member of $\mathcal{G}$ contains both $x$ and $y$.

Proof. Suppose that $\kappa \in D(X)$ and $T \subset X^{2}-\Delta(X)$ are given, with $|T|=\kappa$. Find an open neighborhood $W$ of $\Delta(X)$ such that $\left|T_{0}\right|=\kappa$, where $T_{0}=T-W$. For each $x \in X$ find an open set $G(x) \subset X$ such that $(x, x) \in$ $(G(x))^{2} \subset W$. Let $\mathcal{G}=\{G(x): x \in X\}$.

Conversely, suppose that $X$ satisfies the covering condition above. Given $T$, find $T_{0}$ and $\mathcal{G}$. For each $x \in X$ choose $G(x) \in \mathcal{G}$ with $x \in G(x)$ and let $W=\bigcup\left\{(G(x))^{2}: x \in X\right\}$. Then $T_{0} \subset T-W$ as required to prove that $\kappa \in D(X)$.

(3.2) Proposition. Suppose $(X, \mathcal{T},<)$ is a GO-space such that $\kappa^{+} \in$ $D(X)$. If $c(X) \leq \kappa$ then $d(X) \leq \kappa$.

Proof. From (2.3), we know that either $d(X)=c(X)$, in which case there is nothing to prove, or else $d(X)=c(X)^{+}$. For contradiction, suppose that $d(X)=c(X)^{+}$and $d(X)>\kappa$. Then $c(X)=\kappa$ and $d(X)=\kappa^{+}$.

The construction in Section 2 yields pairwise disjoint collections $\mathcal{U}(\alpha)$ for each $\alpha<\kappa^{+}$. For each $C \in \mathcal{U}(\alpha)$, recall that $C^{\prime}, C^{\prime \prime}$ are the two convex components of $C-\{f(C)\}$ and that $\left.\left.C^{\prime} \subset\right] \leftarrow, f(C)\right]$ and $\left.C^{\prime \prime} \subset\right] f(C), \rightarrow[$.

Let $T=\left\{\left(f(C), f\left(C^{\prime \prime}\right)\right): C \in \mathcal{U}\right\}$. Then $T \subset X^{2}-\Delta(X)$ and $|T|=\kappa^{+}$. Because $\kappa^{+} \in D(X)$, there is an open neighborhood $W$ of $\Delta(X)$ having $|T-W|=\kappa^{+}$. Let $\mathcal{V}=\left\{C \in \mathcal{U}:\left(f(C), f\left(C^{\prime \prime}\right)\right) \in T-W\right\}$. Then $|\mathcal{V}|=\kappa^{+}$.

For each $x \in X$, choose an open set $G(x)$ with $(x, x) \in G(x)^{2} \subset W$. Because $l(X) \leq h l(X)=c(X)=\kappa$, there is a collection $\mathcal{G} \subset\{G(x): x \in X\}$ having $\cup \mathcal{G}=X$ and $|\mathcal{G}|=\kappa$. For each $G \in \mathcal{G}$ define $\mathcal{V}(G)=\{C \in \mathcal{V}$ : $f(C) \in G\}$. Then $\mathcal{V}=\bigcup\{\mathcal{V}(G): G \in \mathcal{G}\}$. Because $|\mathcal{V}|=\kappa^{+}$, for some $G_{0} \in \mathcal{G}$ we have $\left|\mathcal{V}\left(G_{0}\right)\right|=\kappa^{+}$.

Consider two distinct members $C_{1}, C_{2} \in \mathcal{V}\left(G_{0}\right)$ numbered in such a way that $f\left(C_{1}\right)<f\left(C_{2}\right)$. Because $f\left(C_{1}\right) \in G_{0}$ we must have $f\left(C_{1}^{\prime \prime}\right) \notin G_{0}$, for otherwise $\left(f\left(C_{1}\right), f\left(C_{1}^{\prime \prime}\right)\right) \in G_{0} \times G_{0} \subset W$, contrary to $\left(f\left(C_{1}\right), f\left(C_{1}^{\prime \prime}\right)\right) \in$ $T-W$. But then $f\left(C_{2}\right)<f\left(C_{1}^{\prime \prime}\right)$, so that $\left.f\left(C_{2}\right) \in\right] f\left(C_{1}\right), f\left(C_{1}^{\prime \prime}\right)\left[\subset C_{1}\right.$. Because $C_{1}$ and $C_{2}$ are members of a tree and are not disjoint, it follows that $C_{1} \subset C_{2}$ or $C_{2} \subset C_{1}$. Therefore $\mathcal{V}\left(G_{0}\right)$ is linearly ordered by inclusion, and that is impossible by Corollary (2.1).

(3.3) Proposition. Suppose that $X$ is a GO-space with $\kappa^{+} \in D(X)$. Let $J=\{a \in X$ : for some $b>a] a,, b[=\emptyset\}$. If $l(X) \leq \kappa$, then $|J| \leq \kappa$.

Pr o o f. For contradiction, suppose $|J|>\kappa$. Choose $S \subset J$ with $|S|=\kappa^{+}$. For each $a \in S$, choose $b(a)>a$ with $] a, b(a)[=\emptyset$. Let $T=\{(a, b(a))$ : $a \in S\}$. Then $|T|=\kappa^{+}$and $T \cap \Delta(X)=\emptyset$, so that some open neighborhood $W$ of $\Delta(X)$ has $|T-W|=\kappa^{+}$. Let $S_{0}=\{a \in S:(a, b(a)) \in T-W\}$. Then $\left|S_{0}\right|=\kappa^{+}$. 
We claim that $S_{0}$ is a closed discrete subspace of $X$. For any $x \in X$, let $G(x)$ be an open convex subset of $X$ with $G(x) \times G(x) \subset W$. If $a \in$ $G(x) \cap S_{0}$ then $b(a) \notin G(x)$, for otherwise $(a, b(a)) \in G(x)^{2} \subset W$, contrary to $a \in S_{0}$. Therefore $a \in G(x) \cap S_{0}$ implies $\left.\left.G(x) \subset\right] \leftarrow, b(a)[=] \leftarrow, a\right]$, so that $\left|G(x) \cap S_{0}\right| \leq 1$.

But then $S_{0}$ is a closed discrete subspace of $X$ even though $l(X) \leq \kappa$ and $\left|S_{0}\right|=\kappa^{+}$, and that is impossible.

Recall that for any subset $Y$ of a space $X, \psi(Y, X)$ is the least cardinality of a collection $\mathcal{U}$ of open sets such that $\bigcap \mathcal{U}=Y$. For example, the assertion " $\psi\left(\Delta(X), X^{2}\right)=\omega$ " is the statement that $X$ has a $G_{\delta}$-diagonal.

(3.4) Proposition. Let $X$ be a GO-space with $\kappa^{+} \in D(X)$. If $c(X) \leq \kappa$ then $\psi\left(\Delta(X), X^{2}\right)$ is less than or equal to $\kappa$.

Proof. In the light of (3.1) and (3.3), we know that $d(X) \leq \kappa$ and $|J| \leq \kappa$. Let $D$ be a dense subset of $X$ with cardinality $\kappa$. We next define a family of open covers of $X$. There are two cases to consider:

(1) If $a<b<c$ are points of $D$, define $\mathcal{G}(a, b, c)=\{] \leftarrow, b[] a,, c[] b,, \rightarrow[\}$.

(2) If $x \in J$, then there is a unique point $b(x)$ such that $x<b(x)$ and ]$x, b(x)[=\emptyset$. Then define $\mathcal{G}(x)=\{] \leftarrow, b(x)[] x,, \rightarrow[\}$.

In each case, we obtain an open cover of $X$, and the family of covers so obtained has cardinality $\leq \kappa$.

We next show that if $p<q$ are points of $X$, then there is an open cover $\mathcal{H}$ defined as above so that $q \notin \operatorname{St}(p, \mathcal{H})$ and hence $p \notin \operatorname{St}(q, \mathcal{H})$. There are again two cases to consider:

(a) If the interval $] p, q[$ is infinite, we may choose points $a<b<c$ in $D \cap] p, q[$ and then $\operatorname{St}(p, \mathcal{G}(a, b, c))=] \leftarrow, b[$, which does not contain $q$.

(b) If the interval $] p, q[$ is finite, then $p \in J$ so that $\mathcal{G}(p)$ is defined and $\operatorname{St}(p, \mathcal{G}(p))=] \leftarrow, p]$, which does not contain $q$.

For each of the open covers $\mathcal{H}$ defined above, define $W(\mathcal{H})=\bigcup\left\{H^{2}\right.$ : $H \in \mathcal{H}\}$. We thereby obtain $\kappa$-many open subsets of $X \times X$ whose intersection is $\Delta(X)$, as required.

Combining (3.2), (3.3), and (3.4) we obtain:

(3.5) Proposition. Suppose that $X$ is a GO-space and that $\kappa^{+} \in D(X)$. If $c(X) \leq \kappa$, then $\psi\left(\Delta(X), X^{2}\right) \leq c(X)=h l(X) \leq d(X) \leq \kappa$.

If we consider linearly ordered spaces, rather than GO-spaces, then we can add the Lindelöf degree $l(X)$ to the list in (3.5).

(3.6) Proposition. Suppose $X$ is a LOTS with $\kappa^{+} \in D(X)$. If $l(X) \leq \kappa$, then $c(X) \leq \kappa$ and hence $\psi\left(\Delta(X), X^{2}\right) \leq c(X)=h l(X) \leq d(X) \leq \kappa$. 
Proof. If not, then there is a family $\mathcal{U}=\{] x(\alpha), y(\alpha)\left[: \alpha<\kappa^{+}\right\}$of pairwise disjoint, non-empty, open intervals in $X$. Write $A=\left[0, \kappa^{+}[\right.$. Let $T=\{(x(\alpha), y(\alpha)): \alpha \in A\}$. Then $T \cap \Delta(X)=\emptyset$. Because $\kappa^{+} \in D(X),(3.1)$ yields a convex open cover $\mathcal{G}$ of $X$ and a set $T_{0} \subset T$ with $\left|T_{0}\right|=\kappa^{+}$and with the property that if $(x(\alpha), y(\alpha)) \in T_{0}$ then no member of $\mathcal{G}$ contains both $x$ and $y$. Because $l(X)=\kappa$ we may assume that $|\mathcal{G}|=\kappa$.

For each $G \in \mathcal{G}$, let $A(G)=\{\alpha \in A: x(\alpha) \in G\}$. Because $\bigcup \mathcal{G}=X$, we have $A=\bigcup\{A(G): G \in \mathcal{G}\}$. Hence, some $G_{0} \in \mathcal{G}$ has $\left|A\left(G_{0}\right)\right|=\kappa^{+}$. Choose $\alpha, \beta, \gamma \in A\left(G_{0}\right)$ such that $x(\alpha)<x(\beta)<x(\gamma)$. Easy examples show that the order relationship between those points and the associated points $y(\alpha), y(\beta), y(\gamma)$ may vary, so there are cases to consider. If $y(\alpha) \leq x(\gamma)$, then convexity of $G_{0}$ yields $x(\alpha), y(\alpha) \in[x(\alpha), x(\gamma)] \subset G_{0}$, contrary to $\alpha \in$ $A\left(G_{0}\right)$. Hence $x(\gamma)<y(\alpha)$. Next, either $x(\gamma)<y(\beta)$ or else $y(\beta) \leq x(\gamma)$. In the first case we have $x(\alpha)<x(\beta)<x(\gamma)<y(\beta)$ and $x(\alpha)<x(\beta)<x(\gamma)<$ $y(\alpha)$, so that $x(\gamma) \in] x(\beta), y(\beta)[\cap] x(\alpha), y(\alpha)[=\emptyset$, which is impossible. In the second case we have $x(\alpha)<x(\beta)<y(\beta) \leq x(\gamma)$, so that convexity of $G_{0}$ yields $x(\beta), y(\beta) \in[x(\alpha), x(\gamma)] \subset G_{0}$, contrary to $(b(\beta), y(\beta)) \notin W$.

4. Metrization of ordered spaces and small diagonals. In this section, we will use the cardinal function inequalities proved in Section 3 to provide a proof of Theorem (1.2) of the introduction.

(4.1) TheOrem. Suppose $X$ is a Lindelöf LOTS with a small diagonal. Then $X$ is metrizable.

Proof. If $|X| \leq \omega$, there is nothing to prove, so assume that $X$ is uncountable. Then $\omega^{+}=\omega_{1} \in D(X)$. We are given that $l(X)=\omega$ so that (3.6) yields $c(X) \leq \omega$. But then (3.4) yields $\psi\left(\Delta(X), X^{2}\right) \leq \omega$, i.e., that $X$ has a $G_{\delta}$-diagonal. Hence $X$ is metrizable ([L1]).

(4.2) Remark. It is crucial in (4.1) that $X$ be a LOTS and not merely a GO-space, as can be seen from the example of the Sorgenfrey line.

(4.3) Remark. Example (6.2) below is a LOTS with a small diagonal that is not even first countable, let alone metrizable. Thus some hypothesis beyond the existence of a small diagonal is needed in order to make an arbitrary LOTS metrizable.

Because Suslin spaces are a frequently studied class of linearly ordered spaces, it might be worthwhile noting that no Suslin space can have a small diagonal. Indeed, a slightly more general consequence of (3.2) is available, namely:

(4.4) Proposition. Suppose $X$ is a GO-space with a small diagonal. If $c(X)=\omega$, then $X$ is separable. 
It is often the case that diagonal metrization theorems can be proved for countably compact spaces. Among GO-spaces, we have:

(4.5) Proposition. Suppose $X$ is a countably compact GO-space with a small diagonal. Then $X$ is metrizable.

Proof. If we can show that $X$ is paracompact, then it will follow that $X$ is compact and (4.1) will apply to complete the proof. Suppose that $X$ is not paracompact. Then there is a stationary subset $S$ of a regular uncountable cardinal $\kappa$ that embeds in $X$ as a closed subset. Then $S$ inherits both countable compactness and a small diagonal.

Consider $S$ with the topology and ordering inherited from $[0, \kappa[$. For each $s \in S$, let $t(s)$ be the first point of $S$ that is greater than $s$ and let $\sigma$ be the first element of $S$ such that $S_{1}=\{s \in S: s<\sigma\}$ is uncountable. Let $T=$ $\left\{(s, t(s)): s \in S_{1}\right\}$. Then $T \subset S^{2}-\Delta(S)$ has $|T|=\omega_{1}$. Because $\omega_{1} \in D(S)$, (4.1) yields an open convex cover $\mathcal{G}$ of $S$ and an uncountable subset $T_{0} \subset T$ such that if $(s, t(s)) \in T_{0}$ then no member of $\mathcal{G}$ contains both $s$ and $t(s)$. Because $T_{0}$ is uncountable, we may choose a sequence $s(1)<s(2)<\ldots$ of points of $S_{1}$ such that $\left(s(n), t(s(n)) \in T_{0}\right.$ for each $n$. Because $S$ is countably compact, there is a point $p \in S$ such that $p=\lim \{s(n): n \geq 1\}$. Choose $G \in \mathcal{G}$ such that $p \in G$. Then for some $n, s(n) \in G$. Then $s(n)<t(s(n)) \leq$ $s(n+1)$, so that convexity of $G$ forces $\{s(n), t(s(n))\} \subset[s(n), s(n+1)] \subset G$, and that contradicts $\left(s(n), t(s(n)) \in T_{0}\right.$.

5. GO-spaces with H-diagonals. Recall that a space $X$ has an $\mathrm{H}-$ diagonal provided every regular uncountable cardinal $\kappa \leq|X|$ belongs to $D(X)$. It is immediate that:

(5.1) Lemma. If $X$ has a $G_{\delta}$-diagonal, then $X$ has an H-diagonal.

The converse of (5.1) is false, as Example (7.7) shows. In this section we will show that GO-spaces with H-diagonals share two important properties with the class of GO-spaces having $G_{\delta}$-diagonals, namely hereditary paracompactness and first countability. Examples in Section 6 will show that GO-spaces (and even LOTS) with only small diagonals need not have these properties.

(5.2) Lemma. Suppose $Y \subset X$. If $X$ has an H-diagonal, then so does $Y$.

Proof. Suppose $\kappa \leq|Y|$ is a regular uncountable cardinal. Then $\kappa \in$ $D(X)$. If $T \subset Y^{2}-\Delta(Y)$ has cardinality $\kappa$, then $T \subset X^{2}-\Delta(X)$, so that some open neighborhood $W$ of $\Delta(X)$ has $|T-W|=\kappa$. But then $W \cap Y$ is an open neighborhood of $\Delta(Y)$ and $|T-(W \cap Y)|=|T-W|=\kappa$, so that $\kappa \in D(Y)$, as required. 
(5.3) Proposition. Suppose $S$ is a stationary subset of a regular uncountable cardinal $\kappa$. Then $\kappa \notin D(S)$. Consequently, if $X$ is any GO-space with an H-diagonal, then $X$ is hereditarily paracompact.

Proof. Given a stationary $S \subset\left[0, \kappa\left[\right.\right.$, for each $s \in S$, let $s^{\prime}$ be the first element of $S$ that is larger than $s$. Let $T=\left\{\left(s, s^{\prime}\right): s \in S\right\}$. Then $|T|=\kappa$ and an easy Pressing Down Lemma argument shows that $|T-W|<\kappa$ whenever $W$ is an open subset of $X^{2}$ that contains $\Delta(X)$, as required.

Now let $X$ be any GO-space with an H-diagonal. If $X$ is not hereditarily paracompact, then it follows from [EL] that there is an uncountable regular cardinal $\kappa$ and a stationary subset $S \subset[0, \kappa[$ that embeds in $X$. According to (5.2), $S$ must have an H-diagonal, and that is impossible in the light of the first paragraph of this proof.

(5.4) R e mark. Proposition (5.3) is false for GO-spaces that have only a small diagonal, as can be seen from Example (6.3) below.

(5.5) Proposition. Suppose $X$ is a GO-space with an H-diagonal. Then $X$ is first-countable.

Proof. It will be enough to prove that if $p \in X$ and if $[p, \rightarrow$ is not open, then the cofinality of $] \leftarrow, p$ [ is $\omega$, and if $] \leftarrow, p]$ is not open, then the coinitiality of $] p, \rightarrow[$ is also $\omega$. The two proofs are analogous and we will outline the first.

For contradiction, suppose $\kappa=\operatorname{cf}(] \leftarrow, p[)$ is uncountable. Being a cofinality, $\kappa$ is a regular cardinal. Hence $\kappa \in D(X)$. Choose a strictly increasing net $\{x(\alpha): \alpha<\kappa\}$ in $] \leftarrow, p[$ that converges to $p$. Then the net $\{(x(\alpha), x(\alpha+1)): \alpha<\kappa\}$ converges to $(p, p)$ in the space $X^{2}$. Let $T=$ $\{(x(\alpha), x(\alpha+1)): \alpha<\kappa\}$. Then $T \cap \Delta(X)=\emptyset$. However, any neighborhood $W$ of $\Delta(X)$ must have $|T-W|<\kappa$, contrary to $\kappa \in D(X)$.

(5.6) Remark. Proposition (5.5) is not valid for GO-spaces, or even for LOTS, having only a small diagonal, as can be seen from Example (6.2).

Our next result summarizes the cardinal function inequalities from Section 3 for GO-spaces with H-diagonals.

(5.7) Proposition. Let $X$ be a GO-space with an H-diagonal. Then $\psi\left(\Delta(X), X^{2}\right) \leq c(X)=h l(X)=d(X)=h d(X)$.

Proof. We know that $h l(X)=c(X) \leq d(X)=h d(X) \leq c(X)^{+}$because $X$ is a GO-space. Let $c(X)=\kappa$. If $\kappa^{+}>|X|$, then $|X|=\kappa$, so that $\kappa=$ $c(X) \leq d(X) \leq|X|=\kappa$. In addition, $\psi\left(\Delta(X), X^{2}\right) \leq\left|X^{2}\right|=\kappa$. Combining these two inequalities yields the desired conclusion. And if $\kappa^{+} \leq|X|$, then because $X$ has an H-diagonal and $\kappa^{+}$is regular, we have $\kappa^{+} \in D(X)$. The desired conclusion now follows from (3.5). 
(5.8) Remark. As the example of a metrizable discrete LOTS with cardinality $\omega_{1}$ shows, we can have $\psi\left(\Delta(X), X^{2}\right)=\omega<\omega_{1}=c(X)=d(X)=$ $h l(X)$ in (5.7).

If we consider linearly ordered spaces, rather than GO-spaces, then we can add one more cardinal function to the list in (5.8), namely the weight $w(X)$ of $X$.

(5.9) Proposition. Suppose $X$ is a LOTS with an H-diagonal. Then $\psi\left(\Delta(X), X^{2}\right) \leq c(X)=h l(X)=d(X)=h d(X)=w(X)$.

Proof. It will be enough to show that $c(X) \geq w(X)$. Let $\kappa=c(X)$ and let $J(X)=\{a \in X$ : some $b(a)>a$ has $] a, b(a)[=\emptyset\}$. From (3.3) we know that $|J(X)| \leq \kappa$ and from (5.7) we know that $d(X)=\kappa$. But in a LOTS, $w(X)=d(X)+|J(X)| \leq \kappa+\kappa$, as required.

We now consider metrization theory for GO-spaces with H-diagonals. We begin by proving the result announced in (1.6).

(5.10) Proposition. Let $X$ be a locally compact GO-space with an $H$-diagonal. Then $X$ is metrizable.

Proof. By (5.3), $X$ is paracompact. By (4.1), $X$ is locally metrizable. Hence $X$ is metrizable.

As noted in the introduction, Example (7.7) shows that (5.10) cannot be generalized to Čech-complete GO-spaces (or even Čech-complete LOTS) with H-diagonals. However, such GO-spaces do have some special structure, as we will show in our next result.

(5.11) Proposition. Suppose X is a GO-space that is Čech-complete or is a p-space with an H-diagonal. Then $X$ has a dense metrizable subspace.

P r o o f. According to (5.3), $X$ is hereditarily paracompact. According to [vW, Theorem 2.1.7], there is a perfect, order preserving mapping $F$ from $X$ onto a metrizable GO-space $Y$ because $X$ is a paracompact p-space.

For each $y \in Y$, let $C(y)=F^{-1}[\{y\}]$. Each $C(y)$ is a compact convex subset of $X$ and must therefore have the form $C(y)=[a(y), b(y)]$ for some points $a(y) \leq b(y)$ of $X$. Because $X$ has an H-diagonal, it follows that the compact set $C(y)$ is metrizable. Define $M=\bigcup\{\operatorname{Int}(C(y)): y \in Y\}$. Being locally metrizable and paracompact, $M$ is a metrizable subspace of $X$.

Let $\mathcal{W}$ be the family of all convex components of the open set $X-\operatorname{cl}(M)$. Observe that if $W \in \mathcal{W}$ and $y \in Y$, then $W \cap \operatorname{Int}(C(y))=\emptyset$. It follows that no non-empty open subset of $W$ can be finite.

Next observe that the order preserving nature of $F$ guarantees that, with the possible exception of the two end points of $W$, if $x \in W$, then $C(F(x)) \subset W$. In addition, if $p<q<r$ are points of $W$, then it cannot 
happen that $F(p)=F(q)=F(r)$, because that equality would force $q \in$ $W \cap \operatorname{Int}(C(F(q))$. Therefore, if $y \in Y$ and $C(y) \subset W$, then $|C(y)| \leq 2$.

Consider any point $p \in W$ such that $C(F(p)) \not \subset W$. Then $p$ must be an endpoint of $W$, say the left endpoint. We claim that $p \in \operatorname{cl}(W-\{p\})$. For otherwise there would be a convex open neighborhood $N$ of $p$ such that $N \cap(W-\{p\})=\emptyset$. We already know that $[p, \rightarrow[$ is open, because $p$ is the left endpoint of the open set $W$ (and $p \in W$ ). It then follows that $\{p\}$ is open in $X$, and that is impossible, as noted above. Therefore, by removing at most the two endpoints of $W$, we obtain a convex open subset $V \subset W$ such that:

(a) $V$ is dense in $W$;

(b) $V=F^{-1}[F[V]]$;

(c) if $x \in V$ then $\operatorname{Int}\left(F^{-1}[F(x)]\right) \subset V$.

Fix $W \in \mathcal{W}$, find $V \subset W$ as above, and let $f$ be the restriction of $F$ to $V$. Then $f$ is an order preserving, perfect mapping of $V$ onto some subset of $Y$. In addition, $f$ is an irreducible mapping. For suppose $V_{0}$ is an open, convex subset of $V$. Then $V_{0}$ is not finite, so we may choose points $a<b<c$ in $V_{0}$. Then $f^{-1}[f(b)] \subset[a, c] \subset V_{0}$, as required to show that $f$ is irreducible.

Because $f[V]$ is a subset of the metric space $Y$, there is a dense subset $E$ of $f[V]$ such that $E$ is the union of countably many discrete subsets $E(n)$, each closed in the space $Y$. Because $f$ is irreducible, the set $f^{-1}[E]$ is dense in $V$ and hence also in $W$. Write $D(W)=f^{-1}[E]$. For each $n,\left\{f^{-1}[\{e\}]: e \in\right.$ $E(n)\}$ is a closed discrete collection in the space $X$. Because each $f^{-1}[\{e\}]$ consists of at most two points, it follows that the set $D(n)=\bigcup\left\{f^{-1}[\{e\}]\right.$ : $e \in E(n)\}$ is a discrete closed subset of $X$. Therefore, $D(W)$ is a GO-space that is $\sigma$-discrete, and hence $D(W)$ is metrizable.

Therefore, we now know that for each $W \in \mathcal{W}$, there is a dense metrizable subspace $D(W)$ of $W$. Because $M$ and the members of $\mathcal{W}$ are pairwise disjoint open sets in $X$, it follows that $Z=\bigcup\{D(W): W \in \mathcal{W}\} \cup M$ is a dense metrizable subspace of $X$, as required.

6. Preliminary examples. $\mathbb{Z}$ denotes the set of all positive and negative integers and $\mathbb{R}$ denotes the set of real numbers.

(6.1) EXAMPLE. There is a first countable hereditarily paracompact LOTS that does not have a small diagonal.

Proof. Let $S$ be the Sorgenfrey line and let $S^{*}=S \times\{n \in \mathbb{Z}: n \leq 0\}$, ordered lexicographically. Then $S^{*}$ is the smallest LOTS that contains $S$ as a closed subspace. We begin by proving that if $T_{2}$ is any uncountable subset of $\{((x,-1),(x, 0)): x \in S\}$, then for any neighborhood $W$ of the diagonal in $\left(S^{*}\right)^{2}$, we have $W \cap T_{2} \neq \emptyset$. 
Given such a $T_{2}$ let $S_{2}=\left\{x \in S:((x,-1),(x, 0)) \in T_{2}\right\}$. Then $S_{2}$ is an uncountable subset of $S$, so that $S_{2}$ contains a point $p$ with the property that for every $\varepsilon>0$ the set $\left[p, p+\varepsilon\left[\cap S_{2}\right.\right.$ is uncountable. Suppose $W$ is any open neighborhood of the diagonal in $S^{*} \times S^{*}$. Then there is a $\delta>0$ such that $\left(\left[(p, 0),(p+\delta, 0)[)^{2} \subset W\right.\right.$. Choose $\left.x \in\right] p, p+\delta\left[\cap S_{2}\right.$. Then in $S^{*}$ we have $(p, 0)<(x,-1)<(x, 0)<(p+\delta, 0)$, so that $((x,-1),(x, 0)) \in T_{2} \cap W$, as claimed.

To show that $S^{*}$ does not have a small diagonal, let $S_{1}$ be any subset of $S$ with cardinality $\omega_{1}$ and let $T_{1}=\left\{((x,-1),(x, 0)): x \in S_{1}\right\}$. If $S^{*}$ had a small diagonal, there would be an open neighborhood $W$ of the diagonal such that the set $T_{2}=T_{1}-W$ is uncountable. Apply the first part of the proof to show that $T_{2} \cap W \neq \emptyset$, contradicting the definition of $T_{2}$.

(6.2) EXAMPLE. There is a LOTS $X$ with $|X|=\omega_{2}$ and $\omega_{2} \notin D(X)$ and $\omega_{1} \in D(X)$. Thus, $X$ has a small diagonal but not an H-diagonal. In addition, $X$ is not first countable.

Proof. Let $X=\left(\left[0, \omega_{2}[\times \mathbb{Z}) \cup\left\{\left(\omega_{2}, 0\right)\right\}\right.\right.$ with the lexicographic ordering.

(6.3) EXAMPLE. There is a LOTS with a small diagonal that is not paracompact.

Proof. Let $S=\left\{\alpha<\omega_{3}: \operatorname{cf}(\alpha)=\omega_{2}\right\}$. As a subspace of $\left[0, \omega_{3}[, S\right.$ is stationary and therefore is not paracompact. Of course, $S$ is not a LOTS in its natural ordering. However, because $S$ is scattered, a result of Purisch $[\mathrm{P}]$ shows that $S$ can be reordered in such a way that $S$ becomes a LOTS. Because $S$ is not paracompact, we know from (5.3) that $S$ does not have an H-diagonal.

To show that $S$ has a small diagonal, observe that any subspace of $S$ with cardinality $\omega_{1}$ is a closed and discrete subspace of $S$ and is therefore metrizable. Now suppose that $T \subset(S \times S)-\Delta(S)$ has cardinality $\omega_{1}$. Let $S_{0}=\pi_{1}[T] \cup \pi_{2}[T]$ where $\pi_{i}$ are the natural coordinate projections. Then $T \subset\left(S_{0}\right)^{2}-\Delta\left(S_{0}\right)$, so that, $S_{0}$ being metrizable, there is an open subset $U$ of $S^{2}$ with $\Delta\left(S_{0}\right) \subset U$ and with $\left|T-\left(U \cap\left(S_{0}\right)^{2}\right)\right|=\omega_{1}$. Let $W=U \cup\left(S^{2}-\left(S_{0}\right)^{2}\right)$. Then $W$ is open in $S^{2}$, contains $\Delta(S)$, and has $T-W=T-\left(U \cap\left(S_{0}\right)^{2}\right)$.

We note that one can consistently obtain a non-paracompact LOTS with a small diagonal and having cardinality $\omega_{2}$. One begins with an $E\left(\omega_{2}\right)$ set (something that consistently exists), i.e., a stationary subset $S \subset\left[0, \omega_{2}[\right.$ such that $\operatorname{cf}(\alpha)=\omega$ for each $\alpha \in S$, and if $\lambda<\omega_{2}$ has $\operatorname{cf}(\lambda)=\omega_{1}$, then the set $[0, \lambda[\cap S$ is not stationary in $[0, \lambda[$. One proves by induction that $[0, \lambda[\cap S$ is actually metrizable for every such $\lambda$ and it follows that $[0, \lambda] \cap S$ is an open metrizable subspace of $S$ for each $\lambda<\omega_{2}$.

Now consider any set $T \subset S \times S-\Delta(S)$ having cardinality $\omega_{1}$. For some 
$\lambda<\omega_{2}$ we have $T \subset(S \cap[0, \lambda])^{2}-\Delta(S \cap[0, \lambda])$. Because $S \cap[0, \lambda]$ is metrizable, there is an open set $W_{0} \subset(S \cap[0, \lambda])^{2}$ containing $\Delta(S \cap[0, \lambda])$ and having $\left|T-W_{0}\right|=\omega_{1}$. Let $W=W_{0} \cup(] \lambda, \omega_{2}[\cap S)^{2}$. Then $W$ is open in $S^{2}$, contains $\Delta(S)$, and has $|T-W|=\omega_{1}$. Hence $S$ has a small diagonal.

Because $S$ is stationary in $\omega_{2}$, the space $S$ is not paracompact. Therefore, (5.3) implies that $\omega_{2} \notin D(S)$, so that $S$ does not have an H-diagonal. As with the ZFC example given above, there is a reordering of $S$ under which $S$ is a LOTS.

(6.4) ExAmple. There is a Lindelöf GO-space $X$ with a $G_{\delta}$-diagonal (and hence an H-diagonal) that has $c(X)=2^{\omega}$, and a Lindelöf LOTS with a $\sigma$-disjoint base that does not have a small diagonal.

Pr o of. Let $B$ be a Bernstein set in the usual space $\mathbb{R}$ of real numbers, i.e., an uncountable set such that both $B$ and $C=\mathbb{R}-B$ meet every uncountable closed subset of $\mathbb{R}($ cf. $[\mathrm{Ox}])$. Define a GO-space $X$ by retopologizing $\mathbb{R}$ in such a way that all points of $B$ have their usual neighborhoods, and so that each point of $C$ is isolated. Then $X$ is a GO-space with a $G_{\delta}$-diagonal. Let $Y=(B \times\{0\}) \cup(C \times \mathbb{Z})$. Lexicographically ordering $Y$, we obtain a Lindelöf LOTS with a $\sigma$-disjoint base. Because of (4.1), $Y$ cannot have a small diagonal.

\section{A non-metrizable Čech-compete LOTS with an H-diagonal.} In this section, we use a familiar technique of "splitting points" together with a metric space constructed by A. H. Stone [St] to construct the space described in (1.7). We want to thank R. Pol for mentioning Stone's space to us.

(7.1) Construction. Let $Y$ be a subset of a linearly ordered set $X$ where $|Y|$ is an uncountable regular cardinal number. Let

$$
E(Y, X)=((X-Y) \times\{0\}) \cup(Y \times\{-1,1\})
$$

and order $E(Y, X)$ lexicographically. Let $E(Y, X)$ have the usual open interval topology of that order.

(7.2) Proposition. Let $X$ be a metrizable LOTS and suppose $Y \subset X$. Let $\kappa$ be a regular uncountable cardinal. Then the following are equivalent:

(a) $\kappa \in D(E(Y, X))$,

(b) whenever $S \subset Y$ has $|S|=\kappa$, then there is a set $S_{0} \subset S$ such that $\left|S_{0}\right|=\kappa$ and $S_{0}$ is discrete and closed in $X$.

Proof. Write $Z=E(Y, X)$ and define $\pi: Z^{2} \rightarrow X^{2}$ by $\pi((x, i),(y, j))=$ $(x, y)$. Then $\pi$ is continuous, so that the set $\Delta^{*}=\pi^{-1}[\Delta(X)]$ is a $G_{\delta^{-}}$-subset of $Z^{2}$. Note that $\Delta^{*}=\{((x, 0),(x, 0)): x \in X-Y\} \cup\{((y, i),(y, j))$ : $y \in Y$ and $i, j \in\{-1,1\}\}$. 
Suppose (b) holds, and suppose $T \subset Z^{2}-\Delta(Z)$ has regular uncountable cardinality $\kappa$. Either $\left|T \cap \Delta^{*}\right|<\kappa$ or else $\left|T \cap \Delta^{*}\right|=\kappa$. In the first case we replace $T$ by $T-\Delta^{*}$ and complete the proof under the assumption that $T \cap \Delta^{*}=\emptyset$. In the second case, we replace $T$ by $T \cap \Delta^{*}$ and complete the proof under the assumption that $T \subset \Delta^{*}$.

If $T \cap \Delta^{*}=\emptyset$, then the fact that $\Delta^{*}$ is a $G_{\delta}$-subset of $Z^{2}$ yields an open set $W \subset Z^{2}$ that contains the diagonal and has $|T-W|=\kappa$, as required.

Next, suppose that $T \subset \Delta^{*}$. Because $T \cap \Delta(Z)=\emptyset$ we know that $T \subset\{((y, i),(y, j)): y \in Y$ and $\{i, j\}=\{-1,1\}\}$. Then there is a subset $S \subset Y$ of cardinality $\kappa$ such that $T=\{((y,-1),(y, 1)): y \in S\}$ or else $T=\{((y, 1),(y,-1)): y \in S\}$. The two cases are analogous, and we consider only the first.

Because $S$ has cardinality $\kappa$, there is a set $S_{0} \subset S$ that is closed in $X$ and has cardinality $\kappa$. For each $y \in S_{0}$ there is an open interval $I(y)$ in $X$ such that $I(y) \cap S_{0}=\{y\}$. In addition, the open set $X-S_{0}$ is the union of the pairwise disjoint collection $\left\{I_{\alpha}: \alpha \in A\right\}$ of its convex components.

For an open convex set $I \subset X$, define $J(I)=\{(x, i) \in Z: x \in I\}$. Then $J(I)$ is an open convex subset of $Z$. Using that notation, define $V=$ $\bigcup\left\{J\left(I_{\alpha}\right): \alpha \in A\right\}$ and for each $y \in S_{0}$ let $\left.\left.K^{-}(y)=J(I(y)) \cap\right] \leftarrow,(y,-1)\right]$ and $K^{+}(y)=J(I(y)) \cap[(y, 1), \rightarrow[$. The resulting sets cover $Z$, so that the set $W=V^{2} \cup\left(\bigcup\left\{\left(K^{-}(y)\right)^{2} \cup\left(K^{+}(y)\right)^{2}: y \in S_{0}\right\}\right)$ is a neighborhood of the diagonal $\Delta(Z)$ for which $W \cap T=\emptyset$. Thus (a) holds.

Conversely, suppose (a) holds. Let $S \subset Y$ have cardinality $\kappa$. Let $T=$ $\{((y,-1),(y, 1)): y \in S\}$. Then there must be an open neighborhood $W$ of $\Delta(Z)$ in $Z^{2}$ such that $|T-W|=\kappa$. Let $S_{0}=\{y \in S:((y,-1),(y, 1)) \in$ $T-W\}$.

With the exception of endpoints of $Z$ (to be treated separately by analogous arguments), for each $z \in Z$ there are points $(a(z), i(z)),(b(z), j(z)) \in Z$ such that, with $G(z)=](a(z), i(z)),(b(z), j(z))\left[\right.$, we have $(G(z))^{2} \subset W$.

If $x \in X-Y$ let $H(x)=] a(x, 0), b(x, 0)$ [ and if $x \in Y$ let $H(x)=$ ]$a(x,-1), b(x,+1)\left[\right.$. Observe that if $x \in X-Y$ then $H(x) \cap S_{0}=\emptyset$. Further, if $x \in Y$, then $\left|H(x) \cap S_{0}\right| \leq 1$, for otherwise, choose $y \in H(x) \cap S_{0}$ with $y \neq x$. In case $y<x$ we have $(a(x,-1), i(x,-1))<(y,-1)<(y, 1)<(x,-1)$, so that $((y,-1),(y, 1)) \in(G(x,-1))^{2} \subset W$, contrary to $y \in S_{0}$. And if $x<y$ then $((y,-1),(y, 1)) \in(G(x,+1))^{2} \subset W$, again contradicting $y \in S_{0}$. Therefore, $S_{0}$ is a closed and discrete subset of $X$, as required.

(7.3) Corollary. Let $Y \subset X$ where $X$ is any metrizable LOTS. Then $E(Y, X)$ has an H-diagonal if and only if for every regular, uncountable cardinal $\kappa \leq|X|$ and any subset $S \subset Y$ with cardinality $\kappa$, there is a set $S_{0} \subset S$ that is closed in $X$, discrete in itself, and has $\left|S_{0}\right|=\kappa$. 
The next result allows us to recognize when the space $E(Y, X)$ constructed in (7.1) will be metrizable.

(7.4) Proposition. Let $X$ be a metrizable LOTS and let $Y \subset X$ have uncountable regular cardinality. Then the space $E(Y, X)$ of $(7.1)$ is metrizable if and only if $Y$ is the union of countably many subspaces, each of which is discrete in itself.

Pr o of. First suppose that $Z=E(Y, X)$ is metrizable. Let $\mathcal{B}=\bigcup\{\mathcal{B}(n)$ : $n \geq 1\}$ be a $\sigma$-discrete base for $Z$. For each pair $(m, n)$ of natural numbers, let $Y(m, n)$ be the set of all points $y \in Y$ such that for some $B \in \mathcal{B}(m)$ and some $C \in \mathcal{B}(n)$, we have $(y,-1) \in B \subset] \leftarrow,(y,-1)]$ and $(y, 1) \in C \subset$ $[(y, 1), \rightarrow[$. It is easy to check that each $Y(m, n)$ is discrete in itself.

Conversely, suppose $Y$ is the union of countably many subspaces, each discrete in itself. Because $X$ is a metric space, it follows that $Y=\bigcup\{Y(n)$ : $n \geq 1\}$ where each $Y(n)$ is discrete in itself and closed in $X$.

Recall the notation of (7.2). We have a $G_{\delta^{-}}$subset $\Delta^{*}$ of $Z^{2}$ consisting of all points $((x, 0),(x, 0))$ for $x \in X-Y$ together with all points of the form $((y, i),(y, j))$ where $y \in Y$ and $i, j \in\{-1,1\}$. Clearly, $\Delta(X) \subset \Delta^{*}$. In the remainder of the proof, we will define open sets $W(n) \subset Z^{2}$ such that $\Delta(Z)=\Delta^{*} \cap \bigcap\{W(n): n \geq 1\}$. Once that is done, then we will know that $Z$ is a LOTS with a $G_{\delta}$-diagonal and is, therefore, metrizable [L1].

For each $n$ and each $y \in Y(n)$, choose an open interval $I(n, y)$ of $X$ with $I(n, y) \cap Y(n)=\{y\}$. In addition, write the open set $U(n)=X-Y(n)$ as the union of its convex components, say $U(n)=\bigcup\{U(n, \alpha): \alpha \in A(n)\}$. Recall from the proof of (7.2) that for each convex open subset $I \subset X$, we have a convex open subset $J(I)=\{(x, i) \in Z: x \in I\}$ of $Z$.

Define $K(n, y)=J(I(n, y))$ and $V(n, \alpha)=J(U(n, \alpha))$. Then define $L(n,, y,-1)=J(I(n, y)) \cap] \leftarrow,(y,-1)]$ and $L(n, y, 1)=K(n, y) \cap[(y, 1), \rightarrow[$. Let $V(n)=\bigcup\{V(n, \alpha): \alpha \in A(n)\}$. The resulting sets cover $Z$, so that the set $W(n)=(V(n))^{2} \cup \bigcup\left\{(L(n, y,-1))^{2} \cup(L(n, y, 1))^{2}: y \in Y(n)\right\}$ is open in $Z$ and contains $\Delta(Z)$.

Consider any point $p \in \Delta^{*} \cap \bigcap\{W(n): n \geq 1\}$. For contradiction, suppose $p \notin \Delta(Z)$. Then $p=((y, i),(y, j))$ for some $y \in Y$ and $i \neq j$. Choose $n$ so that $y \in Y(n)$. By assumption, $p \in W(n)$. Because $U(n) \cap$ $Y(n)=\emptyset$ we conclude $p \notin(V(n))^{2}$. Hence there is a $y^{\prime} \in Y(n)$ such that $p \in\left(L\left(n, y^{\prime},-1\right)\right)^{2} \cup\left(L\left(n, y^{\prime}, 1\right)\right)^{2} \subset\left(K\left(n, y^{\prime}\right)\right)^{2}$. But then $y \in I\left(n, y^{\prime}\right)$ and therefore $y \in I\left(n, y^{\prime}\right) \cap Y(n)=\left\{y^{\prime}\right\}$, so that $y=y^{\prime}$. But that is impossible because $i \neq j$ guarantees that $((y, i),(y, j)) \notin(L(n, y,-1))^{2} \cup(L(n, y, 1))^{2}$. Therefore $p \in \Delta(Z)$ as required.

Many properties of $E(Y, X)$ are derived from $X$ via the natural projection mapping, and we have: 
(7.5) Lemma. The natural projection mapping $\pi: E(Y, X) \rightarrow X$ given by $\pi((x, m))=x$ is a perfect mapping.

To apply (7.2) to the construction of our example, we must start with a very special completely metrizable LOTS $X$. The following example was constructed by A. H. Stone in his paper [St].

(7.6) EXAMPLE. There is a completely metrizable LOTS X having a subspace $Y$ that satisfies the hypotheses of (7.2).

Proof. Let $D$ be a discrete space with cardinality $\omega_{1}$ and let $Z=D^{\omega}$ carry the product topology. It is proved in [St] that there is a subspace $Y \subset Z$ with the following properties:

(a) $|Y|=\omega_{1}$;

(b) $Y$ is not the union of countably many subspaces, each discrete in itself;

(c) if $S$ is a countable subset of $Y$, then the closure of $S$ in $Y$ is also countable.

Let $X$ be the closure of $Y$ in the complete metric space $Z$. Suppose $S \subset Y$ has uncountable regular cardinality $\kappa$. Then $\kappa=\omega_{1}$. Being a metrizable space, $S$ contains a dense subset $D=\bigcup\{D(n): n \geq 1\}$ such that each $D(n)$ is discrete in itself. For each $n$, let $U(n)$ be the union of all open subsets $V$ of $X$ such that $|V \cap D(n)| \leq 1$. Then $D(n)$ is a relatively closed subset of $U(n)$, and $U(n)$, being open in $X$, is an $F_{\sigma}$-subset of $X$. Write $U(n)=\bigcup\{F(n, m)$ : $m \geq 1\}$ where each $F(n, m)$ is closed in $X$. Let $E(n, m)=F(n, m) \cap D(n)$. Then $E(n, m)$ is closed in $X$ and is discrete in itself. If every $E(n, m)$ were countable, then so would be the set $D$ and then the closure of $D$ in $Y$ would also be countable. But that is not the case, because the closure of $D$ in $Y$ contains the uncountable set $S$. Hence, some set $S_{0}=E(n, m)$ is uncountable and closed in $X$.

We next need show that $X$ is a linearly ordered space in some ordering. But that follows from a theorem of Herrlich ([H]; see also [E, Problem 6.3.2]) stating that any metrizable space with all of its dimensions (dim, ind, Ind) equal to 0 carries the open interval topology of some linear ordering. Because $X$ is a closed subspace of the strongly zero-dimensional metric space $Z$, we have $\operatorname{ind}(X)=\operatorname{Ind}(X)=\operatorname{dim}(X)=0$. Therefore, $X$ is a LOTS in some ordering.

(7.7) ExAMPLE. There is a non-metrizable, Čech-complete LOTS with an H-diagonal.

Proof. Use the metric space $X$ and its subspace $Y$ from (7.6) in the construction of $E(Y, X)$ outlined in (7.1). Apply (7.6) to see that $X$ and $Y$ fit the hypotheses of $(7.2)$ and conclude that $E(Y, X)$ has an H-diagonal. 
Apply the special properties of $Y$ combined with (7.4) to see that $E(Y, X)$ is not metrizable. Apply (7.5) to see that $E(Y, X)$ is a perfect preimage of a complete metric space, i.e., that $E(Y, X)$ is Cech-complete. Being a non-metrizable LOTS, $E(Y, X)$ cannot have a $G_{\delta}$-diagonal [L1].

(7.8) R e mark. In (5.12) we proved that if $X$ is a Cech-complete space (or a p-space) with an H-diagonal, then $X$ has a dense metrizable subspace. In that proof, there was a certain open, metrizable subspace $M$, and $X-$ $\operatorname{cl}(M)$ was the union of open convex components. Closer examination will show that if $X$ is a p-space, then each of those convex components will look very much like a subspace of some $E(Y, X)$ and that if $X$ is Cech-complete, then each of those convex components will be a space of the type $E(Y, X)$, for appropriately chosen $X$ and $Y$.

(7.9) Re mark. Notice that the space $E(Y, X)$ in (7.7) does not have a small diagonal, but does have $c \in D(E(Y, X))$ in the light of $(7.2)$ whenever $\omega_{1}<c$.

8. The Michael line construction, base properties, and diagonal conditions. In this section, $\mathbb{R}, \mathbb{P}, \mathbb{Q}$, and $\mathbb{Z}$ denote, respectively, the sets of real, irrational, and rational numbers, and the set of all integers. When we say "the usual real line $\mathbb{R}$ " we will mean the set $\mathbb{R}$ endowed with its usual Euclidean topology. We will consider certain Michael line constructions using various subsets $S$ of $\mathbb{P}$.

(8.1) Construction. Suppose $S \subset \mathbb{P}$. Let $M(S)$ be the set $\mathbb{Q} \cup S$ topologized so that a basic neighborhood of any point $q \in \mathbb{Q}$ has the form ]$a, b[\cap M(S)$ where $a<q<b$ are rational numbers, and where each point of $S$ is isolated in $M(S)$. Define $M^{*}$ to be the lexicographically ordered set

$$
M^{*}(S)=(\mathbb{Q} \times\{0\}) \cup(S \times \mathbb{Z}) .
$$

Topologize $M^{*}(S)$ by using the usual open interval topology of that lexicographic ordering.

We can summarize the main results of this section as follows: $M^{*}(\mathbb{P})$ is a LOTS with a $\sigma$-disjoint base. Under $\mathrm{CH}$, or under $(\mathrm{MA}+\neg \mathrm{CH}), M^{*}(\mathbb{P})$ does not have an $\mathrm{H}$-diagonal. Whether or not $M^{*}(\mathbb{P})$ has a small diagonal is undecidable in $\mathrm{ZFC}$, as is the question of whether or not $c \in D\left(M^{*}(\mathbb{P})\right)$.

(8.2) Lemma. For each $S \subset \mathbb{P}$, the space $M(S)$ is a closed subspace of the LOTS $M^{*}(S)$, and $M^{*}(S)$ has a $\sigma$-disjoint base of open sets, is first countable, and is hereditarily paracompact.

(8.3) Lemma. For any $S \subset \mathbb{P}$, the following are equivalent:

(a) $M^{*}(S)$ is metrizable;

(b) $M(S)$ is metrizable; 
(c) $S$ is an $F_{\sigma}$-subset of $M(S)$;

(d) There is an $F_{\sigma}$-subset $E$ of the usual real line such that $S \subset E \subset \mathbb{P}$.

Proof. The equivalence of (a) and (b) is found in [L2] and the equivalence of (c) and (d) is clear. That (b) implies (c) follows from the fact that $S$ is open in $M(S)$, so that if $M(S)$ is metrizable, then $S$ must be an $F_{\sigma}$-set. That (c) implies (b) follows from the fact that if $S$ is an $F_{\sigma}$-set in $M(S)$, then $M(S)$ has a $\sigma$-discrete base for its topology.

(8.4) Proposition. Let $S \subset \mathbb{P}$ have uncountable regular cardinality $\kappa$. The following are equivalent:

(a) $\kappa \in D\left(M^{*}(S)\right)$;

(b) For each $S_{1} \subset S$ with $\left|S_{1}\right|=\kappa$, there is a closed subset $S_{0}$ of $M(S)$ with $\left|S_{0}\right|=\kappa$ and $S_{0} \subset S_{1}$;

(c) For each $S_{1} \subset S$ with $\left|S_{1}\right|=\kappa$, there is a subset $S_{0} \subset S_{1}$ with cardinality $\kappa$ and such that the closure of $S_{0}$ in the usual real line is contained in $\mathbb{P}$.

Pr o of. The equivalence of (c) and (b) is straightforward. We prove that (a) and (b) are equivalent. Write $M=M(S)$ and $M^{*}=M^{*}(S)$.

Suppose (a) holds, i.e., $\kappa \in D\left(M^{*}\right)$, and suppose $S_{1} \subset S$ has cardinality $\kappa$. Let $T^{*}=\left\{((x, 0),(x, 1)): s \in S_{1}\right\}$. Because $\left|T^{*}\right|=\kappa$ and $T^{*} \cap \Delta(X)=\emptyset$, there is an open set $W^{*} \subset\left(M^{*}\right)^{2}$ that contains $\Delta\left(M^{*}\right)$ and has $\left|T^{*}-W^{*}\right|=\kappa$. For each $x \in Q$ choose rational numbers $a(x), b(x)$ such that $((x, 0),(x, 0)) \in(](a(x), 0),(b(x), 0)[)^{2} \subset W^{*}$. Let $\left.G(x)=\right] a(x), b(x)[$ for each $x \in Q$. Define $G=\bigcup\{G(x): x \in Q\}$ and let $S_{0}=S_{1}-G$. Then $S_{0}$ is closed in $M$ and if $((x, 0),(x, 1)) \in T^{*}-W^{*}$ then $x \in S_{0}$, so that $\left|S_{0}\right|=\kappa$. Hence (b) holds.

Conversely, suppose (b) holds and suppose $T^{*} \subset\left(M^{*}\right)^{2}-\Delta\left(M^{*}\right)$ has cardinality $\kappa$. Define $\pi:\left(M^{*}\right)^{2} \rightarrow M^{2}$ by $\pi((x, m),(y, n))=(x, y)$. There are two cases to consider:

Case 1: Suppose $\left|\pi\left[T^{*}\right]-\Delta(M)\right|=\kappa$. Because $M$ has a $G_{\delta}$-diagonal, there is an open set $W \subset M^{2}$ that contains the diagonal of $M$ and has $\left|\pi\left[T^{*}\right]-W\right|=\kappa$. For each $x \in \mathbb{Q}$ find rational numbers $a(x), b(x)$ such that $(x, x) \in(] a(x), b(x)[)^{2} \subset W$. Let $\left.G^{*}(x, 0)=\right](a(x), 0),(b(x), 0)[$ and for any $x \in S$ and any $k \in \mathbb{Z}$ let $G^{*}(x, k)=\{(x, k)\}$. Let $W^{*}=\bigcup\left\{\left(G^{*}(x, k)\right)^{2}\right.$ : $\left.(x, k) \in M^{*}\right\}$. Then $\left|T^{*}-W^{*}\right|=\left|\pi\left[T^{*}\right]-W\right|=\kappa$ as required to complete the proof of Case 1 .

Case 2: Suppose $\left|\pi\left[T^{*}\right]-\Delta(M)\right|<\kappa$. Then $\left|\pi\left[T^{*}\right] \cap \Delta(M)\right|=\kappa$. Let $S_{1}=\left\{x \in P:(x, x) \in \pi\left[T^{*}\right]\right\}$ and use (b) to find a set $S_{0} \subset S_{1}$ that is closed in $M$ and has cardinality $\kappa$. For each $z \in \mathbb{Q}$ find rational numbers $a(z), b(z)$ such that $a(z)<z<b(z)$ and $] a(z), b(z)\left[\cap S_{0}=\emptyset\right.$. If $z \in \mathbb{Q}$ define $\left.G^{*}(z, 0)=\right](a(z), 0),(b(z), 0)\left[\right.$ and for $z \in \mathbb{P}$ let $G^{*}(z, k)=\{(z, k)\}$. Letting 
$W^{*}=\bigcup\left\{\left(G^{*}(z, k)\right)^{2}:(z, k) \in M^{*}\right\}$ we obtain an open subset of $\left(M^{*}\right)^{2}$ that contains $\Delta\left(M^{*}\right)$ and has $\left|T^{*}-W^{*}\right|=\left|S_{0}\right|=\kappa$. That completes Case 2.

It is not surprising, given (8.3) and (8.4), that set theory plays a role in deciding whether a given $\kappa$ belongs to $M^{*}(\mathbb{P})$. Recall that a subset $S \subset \mathbb{P}$ is said to be concentrated on $\mathbb{Q}$ provided $|S-U| \leq \omega$ whenever $U$ is an open subset of $\mathbb{R}$ that contains $\mathbb{Q}$.

(8.5) Corollary. $M^{*}(\mathbb{P})$ has a small diagonal if and only if no uncountable subset of $\mathbb{P}$ is concentrated on $\mathbb{Q}$.

Pr o of. In this proof, $\operatorname{cl}(S)$ will denote the closure of a set $S$ in the usual space of real numbers. First, suppose that $M^{*}(\mathbb{P})$ has a small diagonal and yet some uncountable $S \subset \mathbb{P}$ is concentrated on $\mathbb{Q}$. Apply (8.4) to find an uncountable set $S_{0} \subset S$ such that $\operatorname{cl}\left(S_{0}\right) \subset \mathbb{P}$. But then $W=\mathbb{R}-\operatorname{cl}\left(S_{0}\right)$ is an open subset of $\mathbb{R}$ that contains $\mathbb{Q}$, and yet $S-W$ must be uncountable because it contains $S_{0}$. Hence, no uncountable set $S \subset \mathbb{P}$ is concentrated on $\mathbb{Q}$.

Next suppose that no uncountable subset of $\mathbb{P}$ is concentrated on $\mathbb{Q}$. Let $S \subset \mathbb{P}$ be uncountable. Because $S$ cannot be concentrated on $\mathbb{Q}$, there must be an open subset $U$ with $\mathbb{Q} \subset U \subset \mathbb{R}$ and having $|S-U|=\omega_{1}$. Let $S_{0}=S-U$. Then $\operatorname{cl}\left(S_{0}\right) \subset \mathbb{P}$. According to $(8.4), M^{*}(\mathbb{P})$ has a small diagonal.

(8.6) Corollary. Under $C H, M^{*}(\mathbb{P})$ does not have a small diagonal.

Proof. In [M2], Michael uses $\mathrm{CH}$ to construct an uncountable subset of $\mathbb{P}$ that is concentrated on $\mathbb{Q}$. Now invoke (8.5).

(8.7) Proposition. Assume $M A+\neg C H$. If $\kappa$ is an uncountable regular cardinal with $\kappa<c$, then $\kappa \in D\left(M^{*}(\mathbb{P})\right)$. In particular, $M^{*}(\mathbb{P})$ has a small diagonal.

Proof. Let $M=M(\mathbb{P})$ and $M^{*}=M^{*}(\mathbb{P})$. Suppose $T^{*} \subset\left(M^{*}\right)^{2}-$ $\Delta\left(M^{*}\right)$ has cardinality $\kappa$ where $\kappa$ is an uncountable regular cardinal with $\kappa<c$. Let $S$ be the set of all $x \in \mathbb{P}$ such that for some $m, n \in \mathbb{Z}$ and some $y \in \mathbb{R}$, either $((x, m),(y, n)) \in T^{*}$ or $((y, n),(x, m)) \in T^{*}$. Then $|S|=\kappa$ and $T^{*} \subset\left(M^{*}(S)\right)^{2}$. Because every subset of $\mathbb{R}$ with cardinality less than $c$ is a $\mathbb{Q}$-set under MA $+\neg \mathrm{CH}$ (cf. $[\mathrm{Ml}]$ ), it follows from (8.3) that $M^{*}(S)$ is metrizable. Hence there is an open subset $U^{*} \subset\left(M^{*}(S)\right)^{2}$ that contains $\Delta\left(M^{*}(S)\right)$ and has $\left|T^{*}-U^{*}\right|=\kappa$. Because $M^{*}(S)$ is a subspace of $M^{*}(\mathbb{P})$ we may find an open set $V^{*} \subset\left(M^{*}(\mathbb{P})\right)^{2}$ such that $V^{*} \cap\left(M^{*}(S)\right)^{2}=U^{*}$. Define

$$
W^{*}=V^{*} \cup\left\{((x, k),(x, k)) \in\left(M^{*}(\mathbb{P})\right)^{2}: x \in \mathbb{P}\right\} .
$$

Then $W^{*}$ is open in $\left(M^{*}(\mathbb{P})\right)^{2}$, contains $\Delta\left(M^{*}(\mathbb{P})\right)$ and has $T^{*}-W^{*}=$ $T^{*}-U^{*}$. Hence $\kappa \in D\left(M^{*}(\mathbb{P})\right)$. 
(8.8) Proposition. Suppose $c$ is a regular cardinal. Then the following are equivalent:

(a) $c \in D\left(M^{*}(\mathbb{P})\right)$;

(b) $\mathbb{P}$ is the union of some family $\mathcal{L}$ of compact subsets of $\mathbb{P}$ having $|\mathcal{L}|<c$;

(c) $d<c$, where $d$ is the smallest cardinality of a cofinal family of compact subsets of $\mathbb{P}$.

Pr o of. We know from [vD, Theorem 8.2] that $d$ equals the least possible cardinality of a collection of compact subsets whose union is $\mathbb{P}$. Hence (b) and (c) are equivalent. Thus, it will be enough to prove that (a) and (b) are equivalent.

Suppose that (a) holds and, for contradiction, suppose that $\mathbb{P}$ is not the union of any family of compact sets with cardinality less than $c$. Let $\mathcal{C}$ be the family of all compact subsets of $\mathbb{P}$. Then $|\mathcal{C}|=c$, so we may well order $\mathcal{C}$ as $\left\{C_{\alpha}: \alpha<c\right\}$. For each $\alpha<c$ we have $\mathbb{P}-\bigcup\left\{C_{\beta}: \beta \leq \alpha\right\} \neq \emptyset$ because $\mathbb{P}$ is not the union of fewer than $c$ compact sets. Choose a point $x(\alpha) \in \mathbb{P}-\bigcup\left\{C_{\beta}: \beta \leq \alpha\right\}$.

Let $S_{1}=\{x(\alpha): \alpha<c\}$. According to (8.4), because $c \in D\left(M^{*}(\mathbb{P})\right)$ there is a set $S_{0} \subset S_{1}$ with cardinality $c$ and having $\operatorname{cl}\left(S_{0}\right) \subset \mathbb{P}$, where cl denotes closure in the usual real line topology. Let $T(n)=\operatorname{cl}\left(S_{0}\right) \cap[-n, n]$. Each $T(n)$ is a compact subset of $\mathbb{P}$ and for some $m,\left|T(m) \cap S_{0}\right|=c$ because $c$ is regular. Choose $\beta<c$ such that $T(m)=C_{\beta}$. Because $T(m) \cap S_{0}$ has cardinality $c$, there is a point $x(\gamma) \in T(m) \cap S_{0}$ with $\gamma>\beta$. But that is impossible because $\gamma>\beta$ forces $x(\gamma) \in \mathbb{P}-\bigcup\left\{C_{\delta}: \delta \leq \gamma\right\} \subset \mathbb{P}-C_{\beta}$. Therefore, $\mathbb{P}$ can be written as a union of fewer than $c$ compact sets, so that (b) follows from (a).

To show that (b) implies (a), suppose that $\mathcal{L}$ is a family of fewer than $c$ compact sets and that $\mathbb{P}=\bigcup \mathcal{L}$. Suppose $S_{1} \subset \mathbb{P}$ has cardinality $c$. Because $c$ is regular, $|\mathcal{L}|<c$, and $S_{1}=\bigcup\left\{L \cap S_{1}: L \in \mathcal{L}\right\}$, there must be an $L_{1} \in \mathcal{L}$ with $\left|L_{1} \cap S_{1}\right|=c$. Let $S_{0}=L_{1} \cap S_{1}$. Then $\operatorname{cl}\left(S_{0}\right) \subset L_{1} \subset \mathbb{P}$, as required in (8.4) to show that $c \in D\left(M^{*}(\mathbb{P})\right)$.

(8.9) Corollary. If either $\mathrm{CH}$ or $(\mathrm{MA}+\neg \mathrm{CH})$ holds, then $c \notin$ $D\left(M^{*}(\mathbb{P})\right)$.

Proof. If $c=\omega_{1}$ then the assertion follows directly from (8.6). Next suppose that $M A+\neg \mathrm{CH}$ holds. Then $c$ is regular. If $\mathbb{P}$ were the union of fewer than $c$ compact sets, then the intersection of fewer than $c$ dense $G_{\delta}$-subsets of the usual real line $\mathbb{R}$ would be empty, and that is impossible under $\mathrm{MA}+\neg \mathrm{CH}$.

(8.10) Proposition. There is a model of set theory in which $c \in$ $D\left(M^{*}(\mathbb{P})\right)$. 
Proof. In [vD, Theorem 5.1], van Douwen shows that there is a model $\mathcal{M}$ of ZFC in which $c=\omega_{2}$ and $\mathbb{P}$ is the union of $\omega_{1}$ compact subsets of $\mathbb{P}$. Then $c=\omega_{2}$ is certainly regular, so that (8.8) applies to give $c \in$ $D\left(M^{*}(\mathbb{P})\right)$.

(8.11) R e m a rk. As will be proved in (9.4) below, $M^{*}(\mathbb{P})$ does not have an H-diagonal. That can be proved in van Douwen's model in a different way. As noted in (8.10), in $\mathcal{M}$ there is a collection $\mathcal{L}$ of compact subsets of $\mathbb{P}$ with $|\mathcal{L}|=\omega_{1}$ and $\bigcup \mathcal{L}=\mathbb{P}$. Well order $\mathcal{L}$ as $\left\{L_{\alpha}: \alpha<\omega_{1}\right\}$ and for each $\alpha<\omega_{1}$ choose $x(\alpha) \in \mathbb{P}-\bigcup\left\{L_{\beta}: \beta<\alpha\right\}$. Let $S=\left\{x(\alpha): \alpha<\omega_{1}\right\}$ and consider $M^{*}(S)$. It follows from (8.4) that $\omega_{1} \notin D\left(M^{*}(S)\right)$, so that $M^{*}(S)$ does not have an H-diagonal. But then, by $(5.2)$, neither does $M^{*}(\mathbb{P})$. Indeed, $\omega_{1} \notin D\left(M^{*}(\mathbb{P})\right)$, i.e., $M^{*}(\mathbb{P})$ does not have a small diagonal.

9. Additional remarks on $M^{*}(\mathbb{P})$. K. P. Hart pointed out the following extensions of our results in Section 8. In what follows, we will use the fact that the usual space $\mathbb{P}$ of irrational numbers is homeomorphic to the product space $\omega^{\omega}$. For $f, g \in \omega^{\omega}$ we will write $f \leq g$ to mean that $f(n) \leq g(n)$ for every $n$, and $f \leq^{*} g$ to mean that $f(n) \leq g(n)$ for all but finitely many values of $n$. The cardinal functions $b$ and $d$ will be as defined in [vD].

The first result is a translation of (8.4) into the language of $\omega^{\omega}$ and $\leq^{*}$.

(9.1) Proposition. Let $\kappa$ be a regular uncountable cardinal. Then $\kappa \notin$ $D\left(M^{*}(\mathbb{P})\right)$ if and only if there is a subset $A \subset \mathbb{P}$ such that:

(a) $|A|=\kappa$;

(b) For each $f \in \omega^{\omega}$, the set $\left\{a \in A: a \leq^{*} f\right\}$ has cardinality less than $\kappa$.

Proof. First suppose that the set $A \subset \mathbb{P}$ exists, as described above, and for contradiction suppose $\kappa \in D\left(M^{*}(\mathbb{P})\right)$. According to (8.4) there is a set $B \subset A$ with $|B|=\kappa$ and such that the closure of $B$ in the usual real line is a subset of $\mathbb{P}$. Hence there is an open set $U$ containing the rational numbers and having $U \cap B=\emptyset$. But then $B$ is contained in the $\sigma$-compact set $\mathbb{R}-U=\mathbb{P}-U$. As in $[\mathrm{vD}]$ there is a single function $f \in \omega^{\omega}$ such that $B \subset\left\{a \in A: a \leq^{*} f\right\}$. But that is impossible because $|B|=\kappa$ and $\left|\left\{a \in A: a \leq^{*} f\right\}\right|<\kappa$. Therefore $\kappa \notin D\left(M^{*}(\mathbb{P})\right)$.

Conversely, suppose $\kappa \notin D\left(M^{*}(\mathbb{P})\right)$. We will find the required subset $A \subset \omega^{\omega}$. By (8.4) there is a subset $S_{1} \subset \mathbb{P}$ such that $\left|S_{1}\right|=\kappa$ and, whenever $S_{0} \subset S_{1}$ has cardinality $\kappa$, then the closure of $S_{0}$ in the real line meets $\mathbb{Q}$. Fix a homeomorphism $\psi$ from the usual space $\mathbb{P}$ of irrationals onto the product space $\omega^{\omega}$ and let $A=\psi\left[S_{1}\right]$. To verify that $A$ has the required properties, fix any $f: \omega \rightarrow \omega$ and note that the set $F=\left\{g \in \omega^{\omega}: g={ }^{*} f\right\}$ is countable. For each $g \in F$, the set $A(g)=\{a \in A: a(n) \leq g(n)$ for each $n \in \omega\}$ is contained in the compact set $K(g)=\left\{h \in \omega^{\omega}: h(n) \leq g(n)\right.$ for all $\left.n\right\}$. 
Then the set $S(g)=\psi^{-1}[A(g)]$ is a subset of the compact subset $\psi^{-1}[K(g)]$ of $\mathbb{P}$. The special properties of $S_{1}$ guarantee that $|S(g)|$ cannot be greater than or equal to $\kappa$, so that $|A(g)|=|S(g)|<\kappa$. Because the set $F$ is countable and $\kappa$ is regular and $\left\{a \in A: a \leq^{*} f\right\}=\bigcup\{A(g): g \in F\}$, we see that $\left|\left\{a \in A: a \leq^{*} f\right\}\right|<\kappa$ as required.

(9.2) Corollary. Suppose $\kappa$ is a regular uncountable cardinal and $\omega^{\omega}$ contains a subset that is well-ordered by $\leq^{*}$ and has cardinality $\kappa$. Then $\kappa \notin D\left(M^{*}(P)\right.$.

(9.3) Corollary. The cardinal $b$ does not belong to $D\left(M^{*}(\mathbb{P})\right)$.

Proof. According to [vD, Theorem 3.3],

$b=\min \left\{|B|: B \subset \omega^{\omega}, B\right.$ is unbounded, and $B$ is well-ordered by $\left.\leq^{*}\right\}$.

Choose $A \subset \omega^{\omega}$ that is unbounded, well-ordered by $\leq^{*}$ and has cardinality $b$. Fix $f \in \omega^{\omega}$ and consider the set $A(f)=\left\{a \in A: a \leq^{*} f\right\}$. If $A(f)$ were cofinal in $A$, then $A$ would be bounded by $f$. Hence $A(f)$ is not cofinal in $A$, so that because $b$ is a regular cardinal [vD, Theorem 3.1(d)], we have $|A(f)|<b$. According to $(9.1), b \notin D\left(M^{*}(\mathbb{P})\right.$ as claimed.

(9.4) Corollary. In ZFC, $M^{*}(\mathbb{P})$ does not have an H-diagonal.

Proof. Because $b \leq c=\left|M^{*}(\mathbb{P})\right|$ and $b$ is regular [vD, 3.1(d)] and has $b \notin D\left(M^{*}(\mathbb{P})\right)$, we see that $M^{*}(\mathbb{P})$ does not have an H-diagonal.

(9.5) Corollary. If $\kappa$ is regular and larger than $d$, then $\kappa \notin D\left(M^{*}(\mathbb{P})\right)$.

Proof. In $[\mathrm{vD}]$ it is proved that there is a set $D$ of size $d$ that is $\leq$-dominating for ${ }^{\omega} \omega$. Therefore, if $T \subset \mathbb{P}$ has size $\kappa$ then there must be some $x \in D$ such that $\left\{t \in T: t \leq^{*} x\right\}$ has size $\kappa$.

(9.6) Proposition. Suppose B is a finite non-empty subset of $\left[\omega_{1}, c\right]$ that consists of regular cardinals. Then there is a generic extension such that a regular cardinal $\lambda \in\left[\omega_{1}, c\right]$ has $\lambda \notin D\left(M^{*}(\mathbb{P})\right)$ if and only if $\lambda \in B$.

Proof. Index $B=\left\{\kappa_{1}, \ldots, \kappa_{n}\right\}$ where $\kappa_{1}<\ldots<\kappa_{n}$. Let $C=$ $\left\{\left(\alpha_{1}, \ldots, \alpha_{n}\right): \alpha_{i}<\kappa_{i}\right.$ for $\left.1 \leq i \leq n\right\}$ with the coordinatewise partial order. Each countable subset of $C$ is bounded above, so that a theorem of Hechler [Hc] yields a ccc-forcing of size $c$ that introduces a cofinal subset of $\omega_{\omega} \omega$ that is order isomorphic to $C$. For each $x \in C$, let $f_{x}$ be the element of $\omega_{\omega} \omega$ that corresponds to $x$ under that isomorphism.

Fix $i$. The set $C_{i}=\left\{\left(\beta_{1}, \ldots, \beta_{n}\right) \in C: \beta_{j}=0\right.$ if $\left.j \neq i\right\}$ is well-ordered and unbounded, and hence the set $\left\{f_{x}: x \in C_{i}\right\}$ is well-ordered by $\leq^{*}$ and unbounded. By $(9.2), \kappa_{i} \notin D\left(M^{*}(\mathbb{P})\right)$.

Conversely, suppose $\lambda$ is a regular cardinal in $\left[\omega_{1}, c\right]$ such that $\lambda \notin$ $D\left(M^{*}(\mathbb{P})\right)$. According to $(9.1)$ there is a subset $A \subset{ }^{\omega} \omega$ such that $|A|=\lambda$ 
and for each $f \in{ }^{\omega} \omega,\left|\left\{a \in A: a \leq^{*} f\right\}\right|<\lambda$. For contradiction, suppose $\lambda \notin B$. For each $a \in A$ choose $x_{a} \in C$ with $a \leq^{*} f_{x_{a}}$. Write $x_{a}=$ $\left(x_{a}(1), \ldots, x_{a}(n)\right)$. There are three cases to consider.

Case 1: Suppose $\lambda<\kappa_{1}$. Because $\lambda<\kappa_{j}=\operatorname{cf}\left(\kappa_{j}\right)$ for each $j$ and $|A|=\lambda$, there is a $\beta_{j}<\kappa_{j}$ such that $x_{a}(j)<\beta_{j}$ for each $a \in A$. Then $y=\left(\beta_{1}, \ldots, \beta_{j}\right) \in C$ and for each $a \in A, a \leq^{*} f_{x_{a}} \leq^{*} f_{y}$. Therefore $\left\{a \in A: a \leq^{*} f_{y}\right\}$ has cardinality $\lambda$, contrary to our choice of $A$.

Case 2: Suppose there is a $j$ with $j+1 \leq n$ and $\kappa_{j}<\lambda<\kappa_{j+1}$. For $j+1 \leq i \leq n$ choose $\beta_{i}<\kappa_{i}$ such that $x_{a}(i)<\beta_{i}$ for each $a \in A$. For each $\left(\gamma_{1}, \ldots, \gamma_{j}\right) \in\left[0, \kappa_{1}\left[\times \ldots \times\left[0, \kappa_{j}\left[\right.\right.\right.\right.$, let $A\left(\gamma_{1}, \ldots, \gamma_{j}\right)=\{a \in A$ : $x_{a}(i)=\gamma_{i}$ for $\left.1 \leq i \leq j\right\}$. Because $\mid\left[0, \kappa_{1}\left[\times \ldots \times\left[0, \kappa_{j}\left[\mid=\kappa_{j}<\lambda\right.\right.\right.\right.$ and $\lambda$ is regular, we can choose $\gamma_{i}<\kappa_{i}$ such that $\left|A\left(\gamma_{1}, \ldots, \gamma_{j}\right)\right|=\lambda$. Define $y \in C$ by setting $y_{i}=\gamma_{i}$ if $1 \leq i \leq j$ and $y_{i}=\beta_{i}$ if $j+1 \leq i \leq n$. Then for each $a \in A\left(\gamma_{1}, \ldots, \gamma_{j}\right)$ we have $x_{a} \leq y$, so that $a \leq^{*} f_{x_{a}} \leq^{*} f_{y}$ in ${ }^{\omega} \omega$. But then $\left\{a \in A: a \leq^{*} f_{y}\right\}$ has cardinality $\lambda$, contrary to our choice of $A$.

Case 3: Suppose $\kappa_{n}<\lambda$. Then regularity of $\lambda$ guarantees that for some choice of $\gamma_{i}<\kappa_{i}$, the set $A\left(\gamma_{1}, \ldots, \gamma_{n}\right)=\left\{a \in A: x_{a}(i)=\gamma_{i}\right.$ for $\left.1 \leq i \leq n\right\}$ has cardinality $\lambda$. Let $y=\left(\gamma_{1}, \ldots, \gamma_{n}\right)$. Then $a \in A\left(\gamma_{1}, \ldots, \gamma_{n}\right)$ implies $a \leq^{*} f_{x_{a}} \leq^{*} f_{y}$, so that $\left|\left\{a \in A: a \leq^{*} f_{y}\right\}\right|=\lambda$, once again contradicting our choice of $A$.

\section{References}

[A] A. V. Arhangel'skii [A. V. Arkhangel'ski1]], A survey of $C_{\mathrm{p}}$-theory, Questions Answers Gen. Topology 5 (1987), 1-109.

[AT] A. V. Arhangel'skii [A. V. Arkhangel'skiì] and V. V. Tkačuk [V. V. Tkachuk], Calibers and point-finite cellularity of the space $C_{\mathrm{p}}(X)$ and some questions of $S$. Gul'ko and M. Hušek, Topology Appl. 23 (1986), 65-73.

[B1] H. Bennett, On quasi-developable spaces, Gen. Topology Appl. 1 (1971), 253-262.

[B2] -, Point-countability in linearly ordered spaces, Proc. Amer. Math. Soc. 28 (1971), 598-606.

[Bo] C. Borges, On stratifiable spaces, Pacific J. Math. 17 (1966), 1-17.

[vD] E. K. van Douwen, The integers and topology, in: Handbook of Set-Theoretic Topology K. Kunen and J. Vaughan (eds.), North-Holland, Amsterdam, 1984, 111-168.

[E] R. Engelking, General Topology, Heldermann, Berlin, 1989.

[EL] R. Engelking and D. Lutzer, Paracompactness in ordered spaces, Fund. Math. 94 (1977), 49-58.

[Fa] M. Faber, Metrizability in Generalized Ordered Spaces, Math. Centre Tracts 53, Mathematisch Centrum, Amsterdam, 1974.

[Hc] S. Hechler, On the existence of certain cofinal subsets of $\omega_{\omega}$, in: Proc. Sympos. Pure Math. 13, Amer. Math. Soc., Providence, R.I., 1974, 155-173.

[H] H. Herrlich, Ordnungsfähigkeit total-diskontinuierlicher Räume, Math. Ann. 159 (1965), 77-80. 
[H1] M. Hušek, Continuous mappings on subspaces of products, in: Sympos. Math. 17, Academic Press, London, 1976, 25-41.

[H2] - , Topological spaces without $\kappa$-accessible diagonal, Comment. Math. Univ. Carolin. 18 (1977), 777-788.

[JS] I. Juhász and Z. Szentmiklóssy, Convergent free sequences in compact spaces, Proc. Amer. Math. Soc. 116 (1992), 1153-1160.

[L1] D. Lutzer, A metrization theorem for linearly orderable spaces, ibid. 22 (1969), $557-558$.

[L2] -, On generalized ordered spaces, Dissertationes Math. 89 (1971).

[M1] E. Michael, The product of a normal space and a metric space need not be normal, Bull. Amer. Math. Soc. 69 (1963), 375-376.

[M2] - Paracompactness and the Lindelöf property in finite and countable Cartesian products, Compositio Math. 23 (1971), 199-214.

[Ml] A. Miller, Special subsets of the real line, in: Handbook of Set-Theoretic Topology, K. Kunen and J. Vaughan (eds.), North-Holland, Amsterdam, 1984, 201-234.

[Ok] A. Okuyama, On metrizability of M-spaces, Proc. Japan Acad. 40 (1964), 176179.

[P] S. Purisch, Scattered compactifications and the orderability of scattered spaces, Proc. Amer. Math. Soc. 95 (1985), 636-640.

[S] V. E. Šneider [V. E. Shneı̆der], Continuous images of Suslin and Borel sets. Metrization theorems, Dokl. Akad. Nauk SSSR 50 (1945), 77-79 (in Russian).

[So] R. Sorgenfrey, On the topological product of paracompact spaces, Bull. Amer. Math. Soc. 53 (1947), 631-632.

[St] A. Stone, On $\sigma$-discreteness and Borel isomorphism, Amer. J. Math. 85 (1963), 655-666.

[vW] J. van Wouwe, GO-Spaces and Generalizations of Metrizability, Math. Centre Tracts 104, Mathematisch Centrum, Amsterdam, 1979.

[Zh] H. X. Zhou, On the small diagonals, Topology Appl. 13 (1982), 283-293.

Mathematics Department

Texas Tech University

Lubbock, Texas 79409

U.S.A.

E-mail: graddir@math.ttu.edu
Mathematics Department College of William and Mary Williamsburg, Virginia 23187 U.S.A.

E-mail: djlutz@mail.wm.edu

Received 22 January 1996;

in revised form 13 October 1996 